\title{
A Note on Simulation of Claim Activity for Use in Aggregate Loss Distributions
}

\author{
by Daniel K. Lyons
}


A NOTE ON SIMULATION OF CLAIM ACTIVITY FOR USE IN AGGREGATE LOSS DISTRIBUTIONS

\begin{abstract}
Aggregate loss distributions have been used in a number of different applications over the last few years. These applications have usually focused on the distribution of losses at ultimate or final values and have not studied how losses move to ultimate values over time. The approach outlined in this note models claim activity through the use of transition matrices. Individual claim activity is then incorporated into an aggregate loss simulation model to determine a number of distributions of interest.
\end{abstract}


This note will present an overview of how to determine the distribution of paid, case, and incurred but not reported (IBNR) losses over time in a manner consistent with the determination of aggregate loss distributions. The method is based on determining severity distributions for both paid and case incurred losses at different valuations, determining transition matrices to model claim changes over time, and simulating many years of claim activity. This method may require much computer time and, if it is to be company specific, detailed loss stratification data. While these requirements may by burdensome the method aiso permits an analysis of the distribution of loss development factors and of run off ratios.

In 1988 Hayne outlined an approach' using collective risk theory to measure the variability of loss reserves. The approach used in this note is an application of the use of collective risk theory such that claim development may be introduced into the process.

When the Insurance Services Office (ISO) prepares a review of increased limits factors they track the severity distribution over time. This is done because ISO is interested in the distribution of losses at their ultimate values. ISO's supplementary exhibits show triangles of pareto parameters obtained from fitting curves to accident year case incurred losses at various valuations. These fits and the relationship between the curves are used to determine the final severity curve upon which indicated increased limits factors are based. This material generally shows the average size of loss increases as the accident year matures.

Severity distributions are needed in determining aggregate loss distributions. Much has been written about the use of aggregate distributions and there are a few methods to use to calculate an aggregate distribution. ${ }^{2,3}$ In a recent paper ${ }^{4}$ Bear and Nemlick use aggregate loss distributions to quantify the expected impact of swing rated reinsurance contracts. In 1980 Patrik and John ${ }^{5}$ used the notion of supporting surplus as measured by the use of an aggregate loss distribution to determine the appropriate load for working cover reinsurance treaties. All of these methods use severity distributions at ultimate or final values. ISO uses severity distributions at 
different valuations in their increased limit reviews but do not measure how individual claims change from one valuation to another. Transition matrices could be used to model this activity. In the formulation of the algorithm used in this paper I am using the severity distribution format as used by Heckman and Meyers (probability of loss in certain intervals is specified, the loss within an interval is uniform). Appendix A sheets one and five show the severity distributions for paid and incurred losses at twelve, twenty four, thirty six, and forty eight month valuations. The average loss is shown at the bottom of each column. Sheets two through four and six through eight show the transition matrices to go from one valuation to the next. Since I am using severity distributions consisting of twenty intervals each transition matrix is twenty by twenty. The second column in sheet 2 , the column labeled " 0 ", shows the movement of claims in the first loss interval ( $\$ 0$ to $\$ 5,000$ ) at twelve months to other loss intervals at twenty four months. In this example $45 \%$ of claims remain in the first interval. Twenty five percent of claims move up an interval $(\$ 5,000$ to $\$ 10,000), 15 \%$ move up two intervals, $10 \%$ three intervals, and $5 \%$ four intervals. Other columns show how losses in other intervals are expected to move during the course of the development period. You will note; entries in each column sum to one, amounts beneath the diagonal represent positive development (claims get larger), and entries above the diagonal represent negative development (claims get smaller). In terms of matrix notation if $S_{1}$ is the severity vector at the first valuation and $T_{12}$ is the first to second valuation transition matrix then $S_{2}$, the severity distribution at the second valuation, equals $T_{12} \cdot S_{1}$. This can be extended so that $S_{3}=T_{23} \cdot S_{2}, S_{4}=T_{34} \cdot S_{3}$ and so on. The ultimate severity distribution can be obtained from the initial severity distribution at twelve months and all the transition matrices.

This approach can be used for paid losses as well as case incurred losses. If paid and incurred transactions are used from the same set of losses you should be able to produce the same ultimate severity distribution in both instances. The illustrative paid and case incurred material 
(strictly hypothetical and not based on any data set) contained in Appendix A produce roughly the same severity distribution at forty eight months.

These initial severity distributions and transition matrices are used to model the paid and case incurred activity on a claim by claim basis. This routine is then used in a simulation program to calculate an aggregate loss distribution. The final aggregate loss distribution is similar to one produced using the Heckman - Meyers algorithm. This approach extends the aggregate loss distribution over development and payment periods in a way which is consistent with the ideas underlying the collective risk model.

To illustrate this I used the following algorithm to produce aggregate losses:

1. Randomly select the number of claims for a year from a negative binomial distribution with mean equal to 126 (approximately) and variance 378 . The mean number of claims was selected so that the expected ultimate loss amount is about $\$ 5,000,000$.

2. For each claim randomly select a report lag from a poisson distribution with mean equal to one half. If the lag is greater than two, cap the lag at two. This was done so that all years would be at ultimate values at the end of six development years. For purposes of simplification the initial severity distributions and transition matrices do not vary as a function of lag. In this example if the lag for a claim is one year the twelve month severity distribution is used as the twenty four month severity distribution and all transition matrices are adjusted accordingly. In practice the initial severity distributions and transition matrices would likely vary as a function of lag because claims which are reported later usually have higher average values.

3. For each claim randomly select a loss interval from the case incurred loss severity distribution at twelve months. Within the interval randomly select a loss amount on the assumption that losses are uniformly distributed in the interval. This is the value of the claim at twelve months. 
4. For each claim at twelve months enter the appropriate column of the twelve to twenty four month transition matrix (based on the loss interval) and randomly select a loss interval for the twenty four month valuation (determined by the row). If the loss interval does not change use the twelve month loss value at the value of the claim at twenty four months. If the loss interval changes randomly select a loss amount on the assumption that losses are uniformly distributed in the new interval.

5. Repeat step four for the other development periods until the claim is at ultimate. This produces a series of case incurred claim amounts for an individual claim at different loss valuations.

6. When the final or ultimate loss interval is determined work backwards using the paid transition matrices and paid severity distributions to determine the payment history for the claim. For example, suppose a claim is in the tenth loss interval at development period four (this is ultimate). It is possible to determine what loss intervals the claim could have been in at period three (i.e., those columns that have a non-zero entry in the tenth row of the transition matrix) and to randomly select a period three loss interval based on the relevant transition matrix and the period three paid severity distribution. That is, the probability of being in the tenth interval at period four equals $\Sigma\left(t_{10 j} \cdot s_{j}\right), j=1$ to 20 where $s_{j}$ is the probability of being in the $j^{\text {th }}$ interval of the period three severity distribution and $t_{10 j}$ is the tenth row of the period three to four transition matrix. Randomly assign a column $\mathrm{j}$ based on the ratio of $t_{10 \mathrm{j}} \cdot \mathrm{s}_{\mathrm{j}}$ to $\Sigma\left(\mathrm{t}_{10 \mathrm{j}} \cdot\right.$ $\left.s_{j}\right)$.

7. When all payment values for a claim are determined accumulate the paid and case incurred values and repeat steps two through six until all claims as specified in step one are finished.

8. Repeat steps one through seven for the desired number of simulation years. 
I ran this procedure for 10,000 years using the material in Appendix A. The table below shows the average paid and case incurred values generated by the simulation:

$\begin{array}{crc}\text { Valuation } & \text { Paid Loss } & \begin{array}{c}\text { Case } \\ \text { Incurred } \\ \text { Loss }\end{array} \\ 12 & 697,224 & 1,160,996 \\ 24 & 1,768,930 & 2,729,345 \\ 36 & 3,289,626 & 4,095,227 \\ 48 & 4,486,742 & 4,783,310 \\ 60 & 4,927,417 & 4,982,895 \\ 72 & 5,010,529 & 5,010,529\end{array}$

More importantly I accumulated various distributions about average values. Rather than show tables of the resultant distributions I will illustrate them graphically. (The program output can be used to calculate means, variances, deciles, etc.) Exhibits $A$ through $G$ show the graphs of a number of distributions.

Exhibit A, Sheet two shows the distribution of losses at ultimate values. I have labeled this "Outstanding Losses at Time $0^{\prime \prime}$ because it represents the a priori distribution ' of loss before any experience has been registered. This graph was prepared using losses at their ultimate values after the simulation had worked through all of the transition matrices. Using the accumulated loss arrays by year it is also possible to determine the distribution of outstanding losses (case outstanding and IBNR) at the end of any valuation. The distribution of outstanding losses is obtained by subtracting paid losses from ultimate losses. Exhibit $\mathrm{A}$, Sheets three through five shows the distribution of outstanding losses at the end of the first, second, and third valuation respectively. Exhibit $A$, Sheet one shows these distributions on the same graph. This illustrates the reduction in average outstanding loss as well as variance over time. It is important to realize that these distributions are on an a priori basis. To determine the variability of reserves given a 
particular amount of reported losses at a specified valuation it would be necessary to determine the outstanding loss distribution on a conditional basis. If variability arouse only from claim counts and the first valuation severity distribution (i.e., there were no IBNR claims or case development) the conditional variance of outstanding losses at the first valuation would be zero.

Exhibit B shows similar graphs for IBNR reserves. These distributions were calculated by subtracting case incurred losses from ultimate losses. Exhibit B, Sheet one, as in Exhibit A, Sheet one, shows the reduction in average IBNR reserves and variance over time.

Exhibits $C$ and $D$ were determined from the accumulated loss arrays too. These graphs show the distribution of incremental (calendar year) paid and case reported losses respectively for a variety of valuations.

Exhibits $E$ and $F$ show the distribution of paid and case incurred loss development factors. The substantial reduction in loss development factor variance as losses mature is particularly noticeable in the sheet one of both exhibits. This type of analysis could be helpful in establishing credibility standards for development factors or to help select the underlying curve to use to model loss development factors for other variability of loss reserve approaches. ${ }^{6,7}$

Exhibit $G$ shows the distribution of run off ratios of loss reserves as of twelve months. I used case incurred loss development factors to estimate ultimate losses and calculated the run off ratio by dividing ultimate losses less paid losses at twelve months by estimated ultimate losses at twelve months less paid losses at twelve months. I did not allow for sufficient room in the program output to show the tail of the distribution - in this example it appears there is continued risk of adverse run off in excess of $50 \%$ of carried reserves. This type of analysis might be used to test different IBNR reserving methods under different claim department reserving practices. ${ }^{8}$

I have tried to outline a straightforward approach that might be used to help quantify the variability of a number of different reserve amounts or loss development measures. I am aware that specifying the transition matrices for different development periods on both a paid and case 
basis could be time consuming and that once accomplished the simulations could take a great deal of computer time. However, there is no substitute for data and it is appealing that such transition matrices could be tailored to individual claim department practices and empirical severity distributions. In addition computer performance continues to improve making large simulation exercises more practical.

I am also aware that this method does not address parameter risk. This is an important source of risk and the variance indications obtained from this approach should be viewed accordingly.

\section{REFERENCES}

1 Hayne, R., "Application of Collective Risk Theory to Estimate Variability in Loss Reserves", 1988 Casualty Actuarial Society Discussion Paper Program, p. 275.

${ }^{2}$ Heckman, P. and Meyers, G., "The Calculation of Aggregate Loss Distributions from Claim Severity and Claim Count Distributions", Proceedings of the Casualty Actuarial Society, LXX (1983), p. 22.

3 Panjer, H. "Recursive Evaluation of a Family of Compound Distributions", ASTIN Bulletin, v. 12 (1981),p. 22.

${ }^{4}$ Bear, R. and Nemlick, K. "Pricing the Impact of Adjustable Features and Loss Sharing Provisions of Reinsurance Treaties", Proceedings of the Casualty Actuarial Society, LXXVII (1990), p. 60.

SPatrik, G. and John, R., "Pricing Excess of Loss Casualty Working Cover Reinsurance Treaties", Casualty Actuarial Society 1980 Discussion Paper Program, p. 399. 
6Kelly, M. "Practical Loss Reserving Method with Stochastic Development Factors", 1992 Casualty Actuarial Society Discussion Paper Program, p. 355.

${ }^{7}$ Hayne, R., "An Estimate of Statistical Variation in Development Factor Methods", Proceedings of the Casualty Actuarial Society LXXII (1985), p. 25.

${ }^{8}$ Stanard, J. "A Simulation Test of Prediction Errors of Loss reserve Estimation Techniques", Proceedings of the Casualty Actuarial Society LXXII (1985), p. 124. 
Exhibit A

Sheet 1

\section{Aggregate Outstanding Losses}

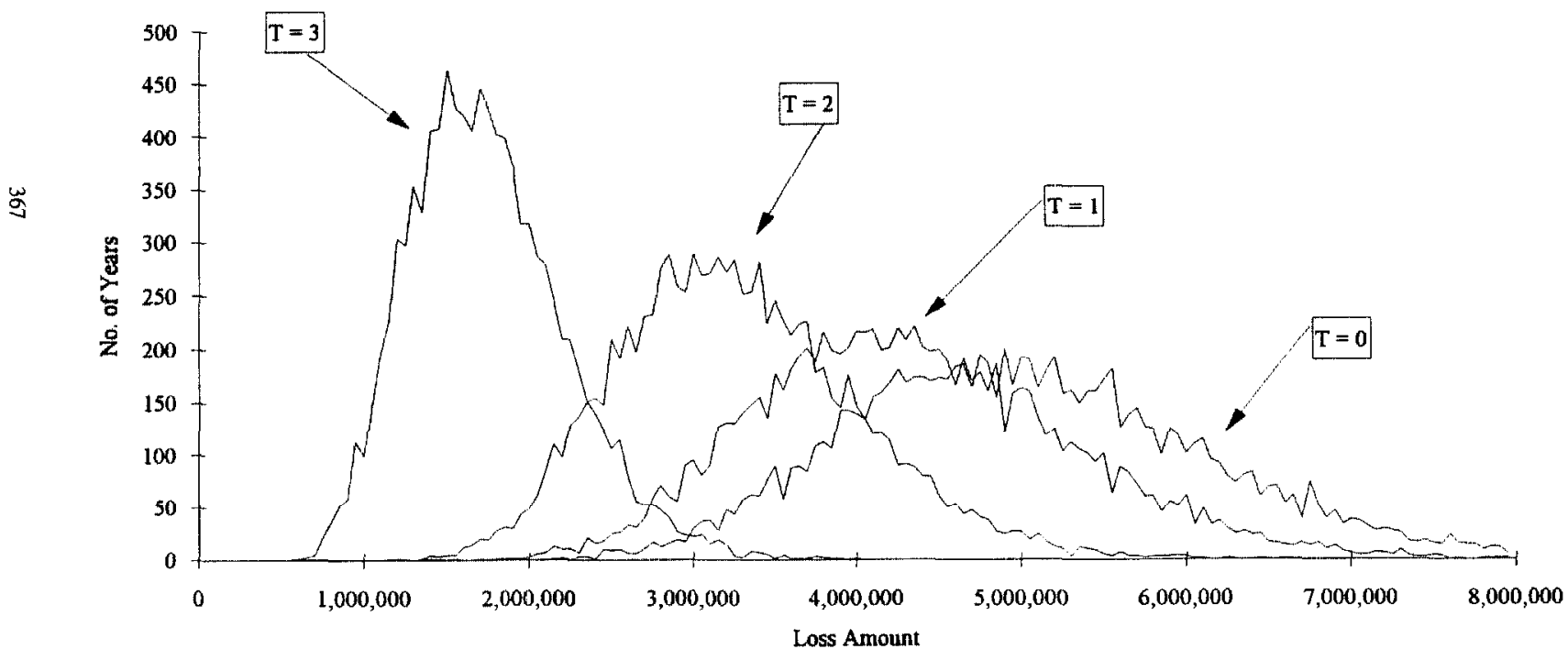


Exhibit A

Sheet 2

Aggregate Outstanding Losses at Time $=0$

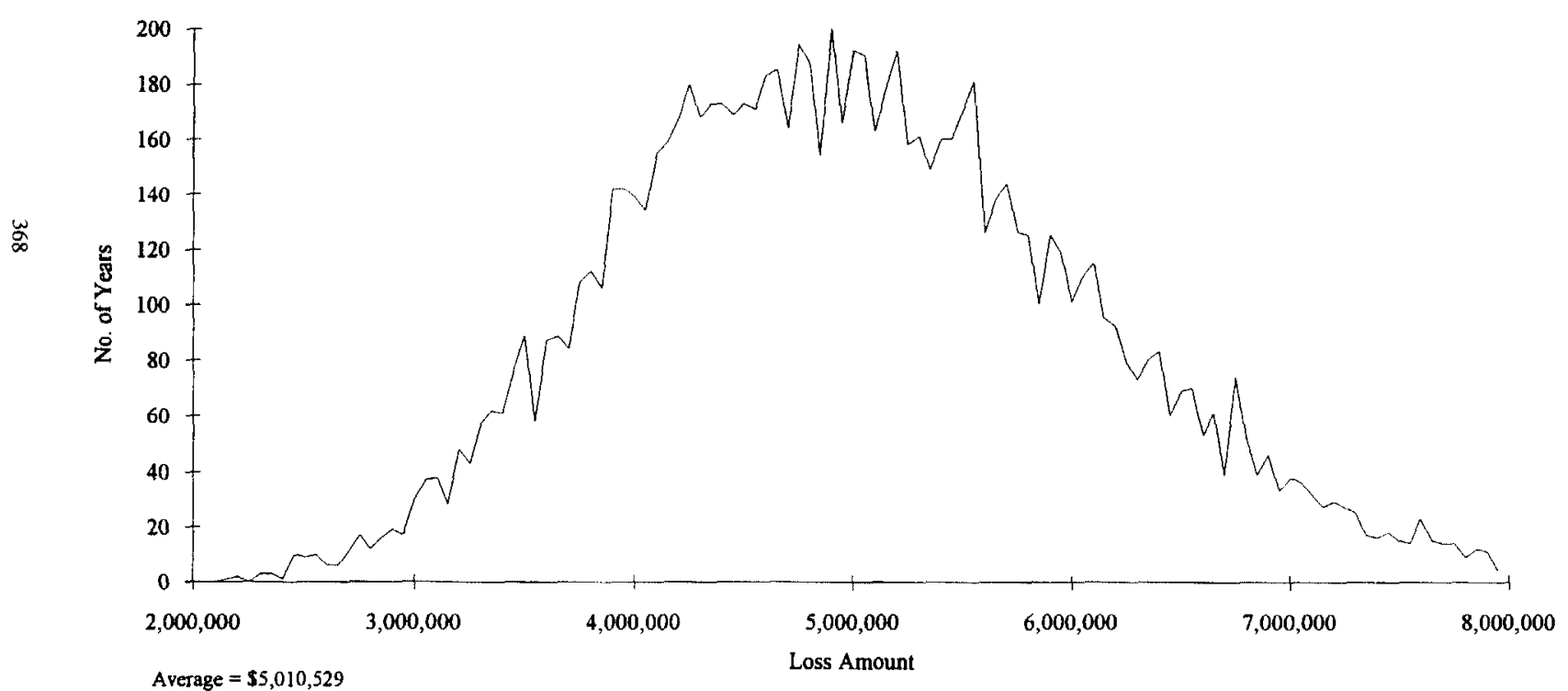


Exhibit A

Sheet 3

Aggregate Outstanding Losses at Time $=1$

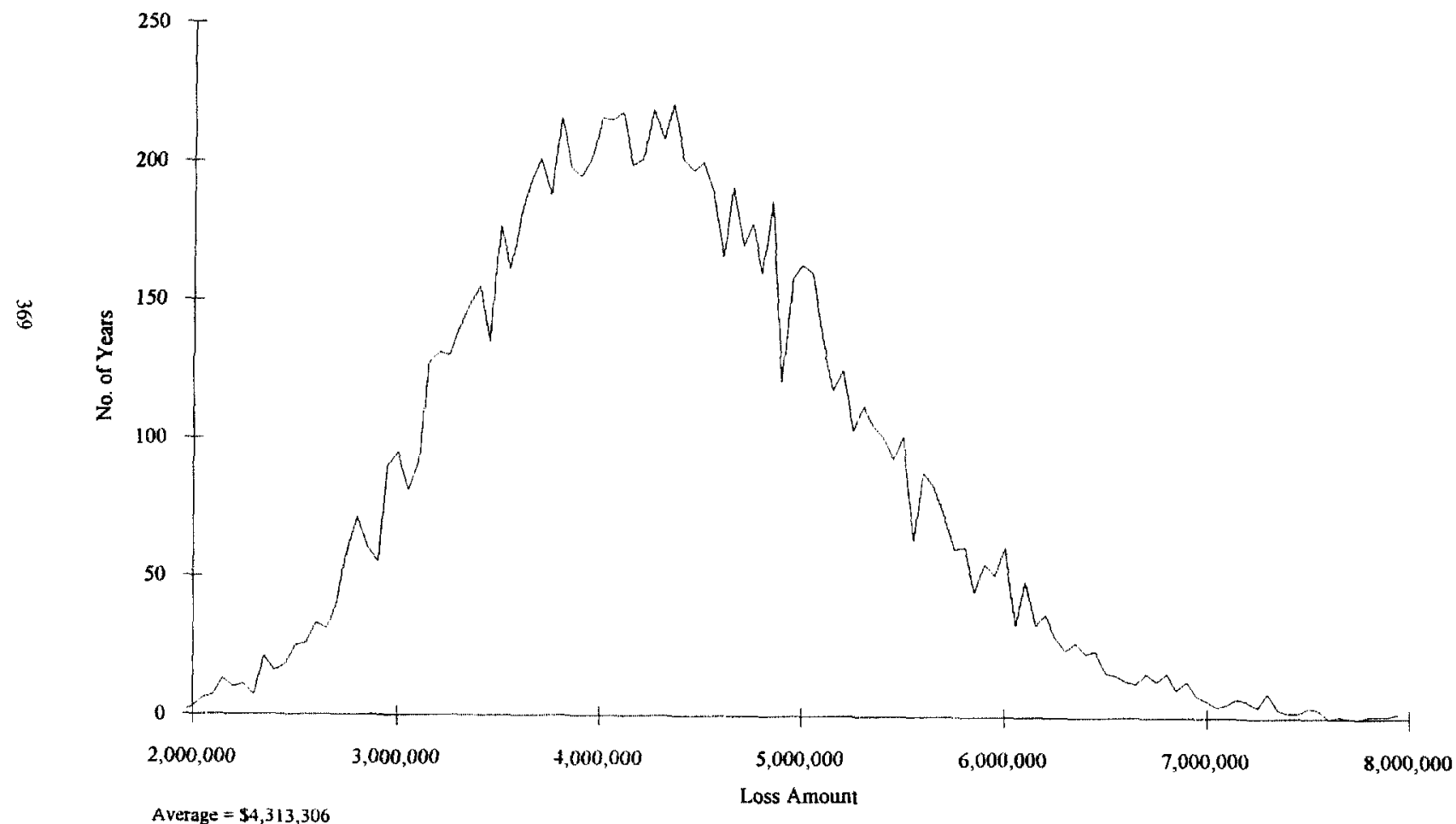




\section{Aggregate Outstanding Losses at Time $=2$}

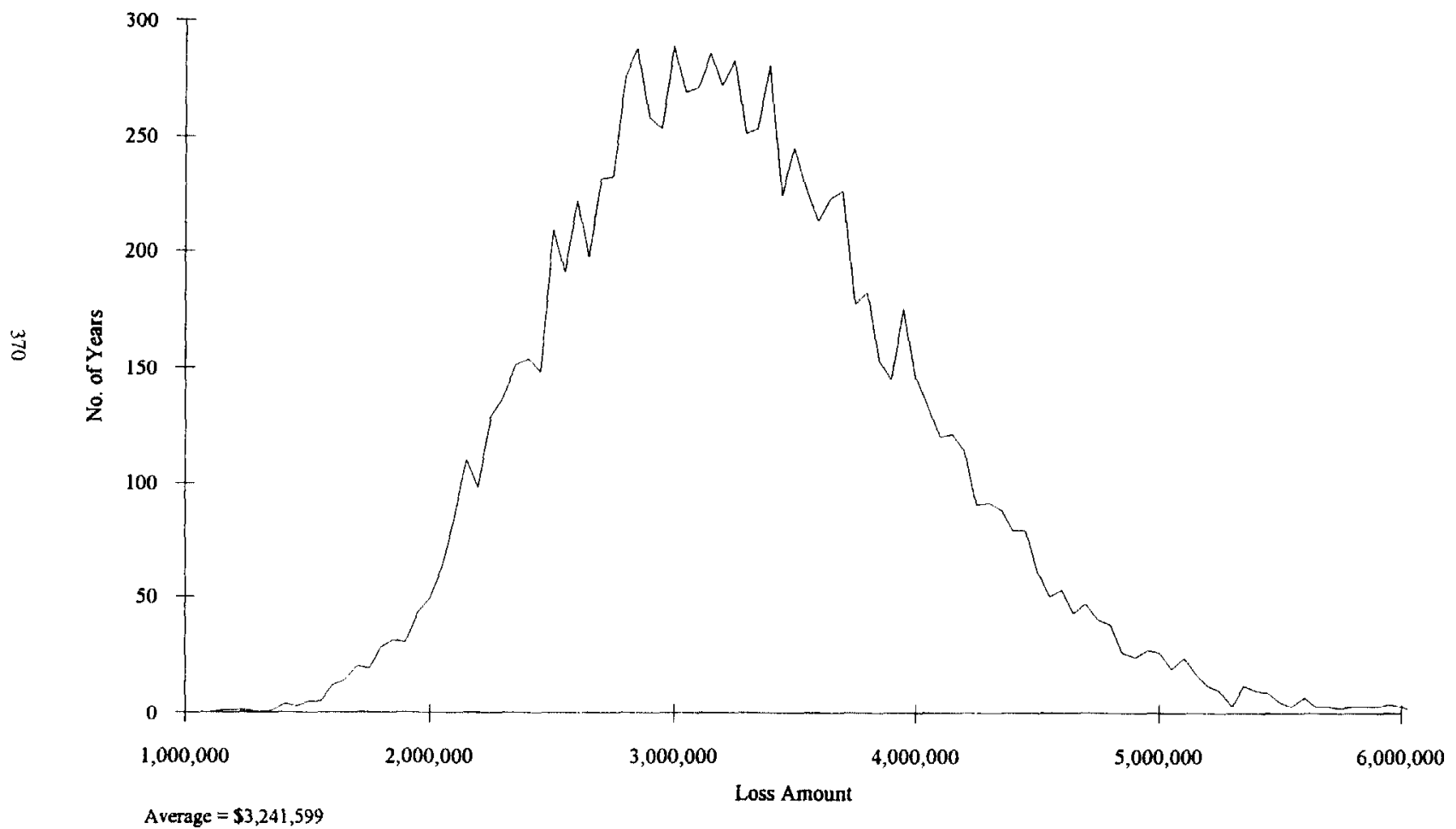


Exhibit A

Sheet 5

Aggregate Outstanding Losses at Time $=3$




Exhibit B

Sheet 1

Aggregate IBNR Losses

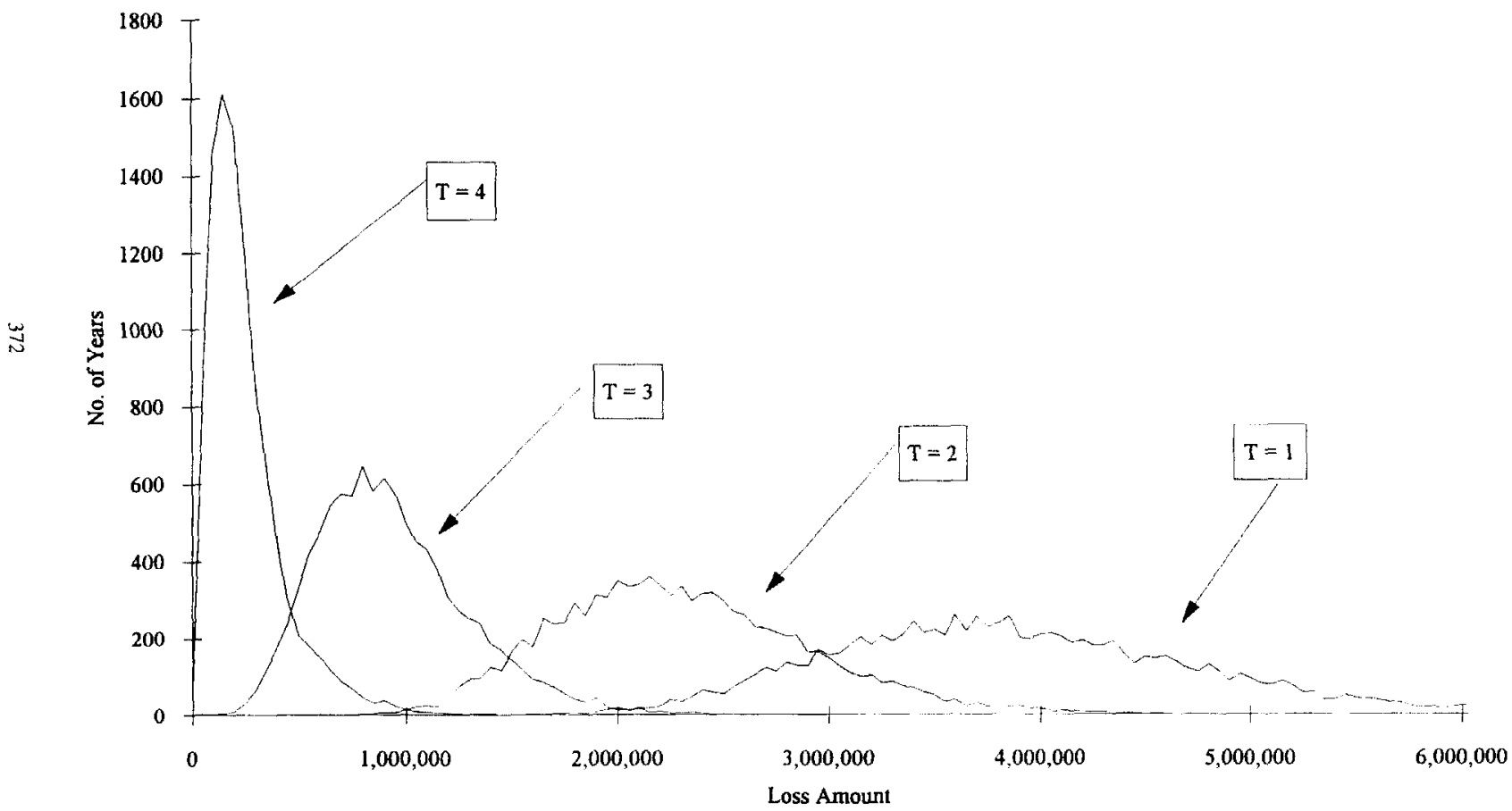


Aggregate IBNR Losses at Time $=1$

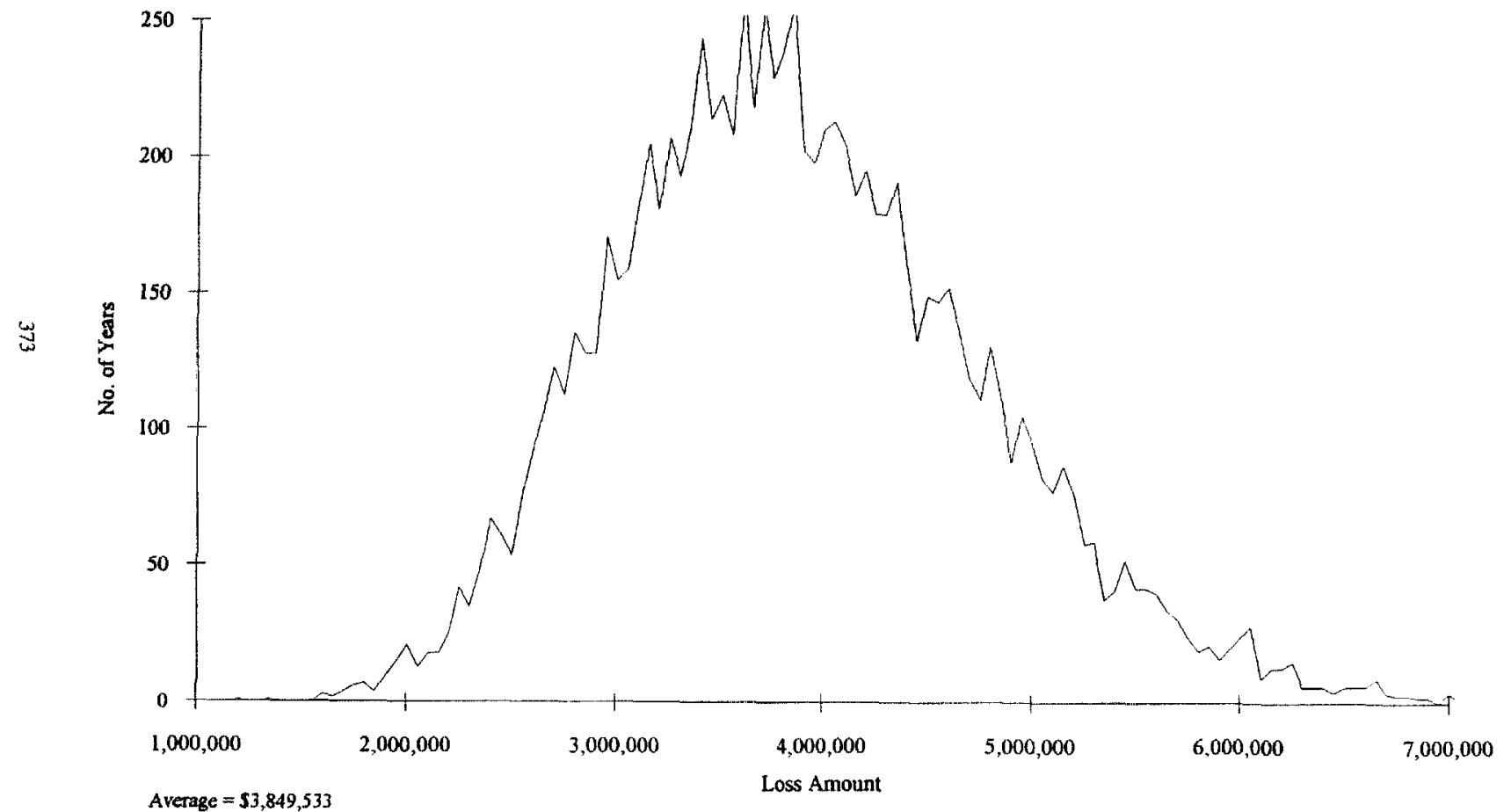


Aggregate IBNR Losses at Time $=2$

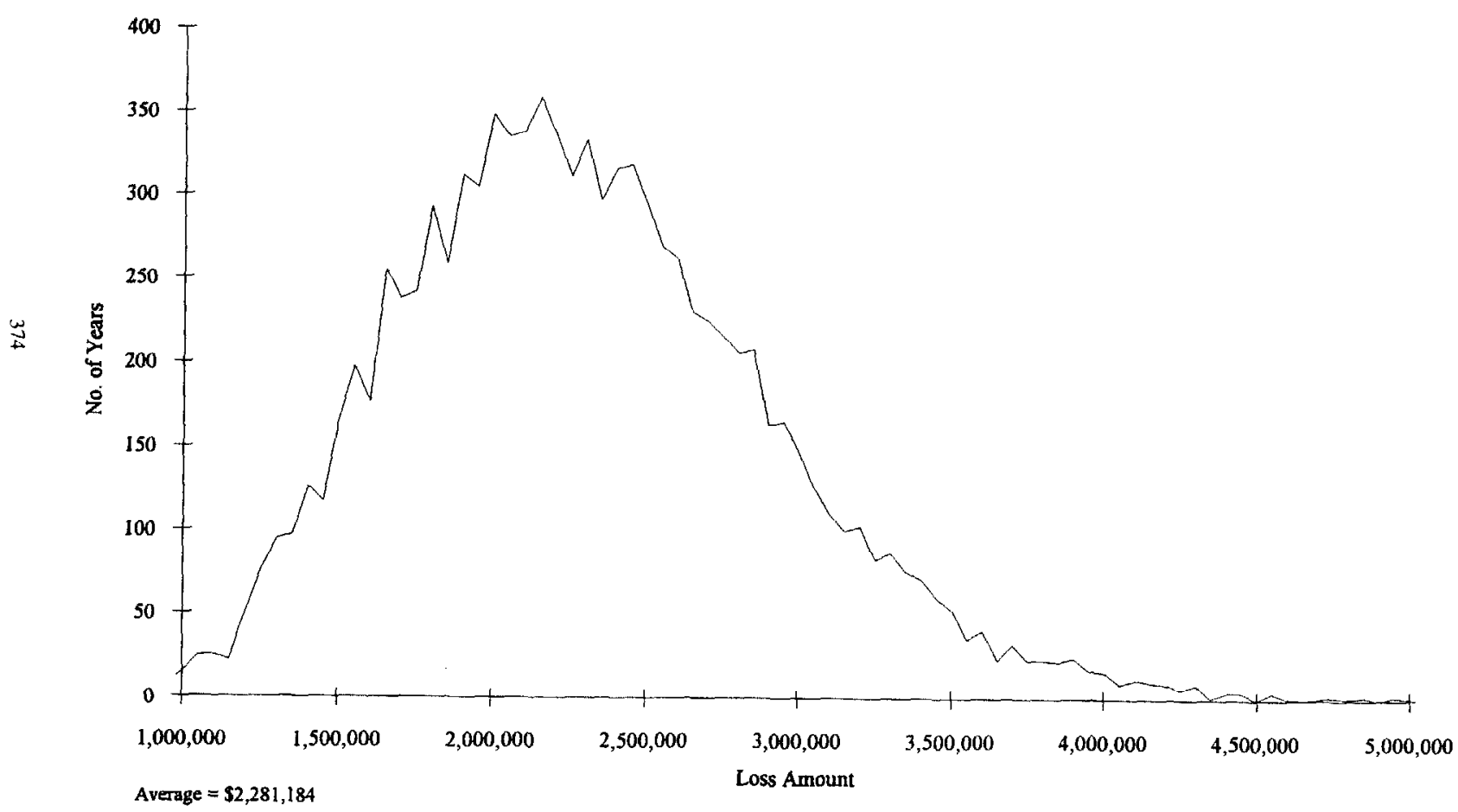


Exhibit B

Sheet 4

Aggregate IBNR Losses at Time $=3$

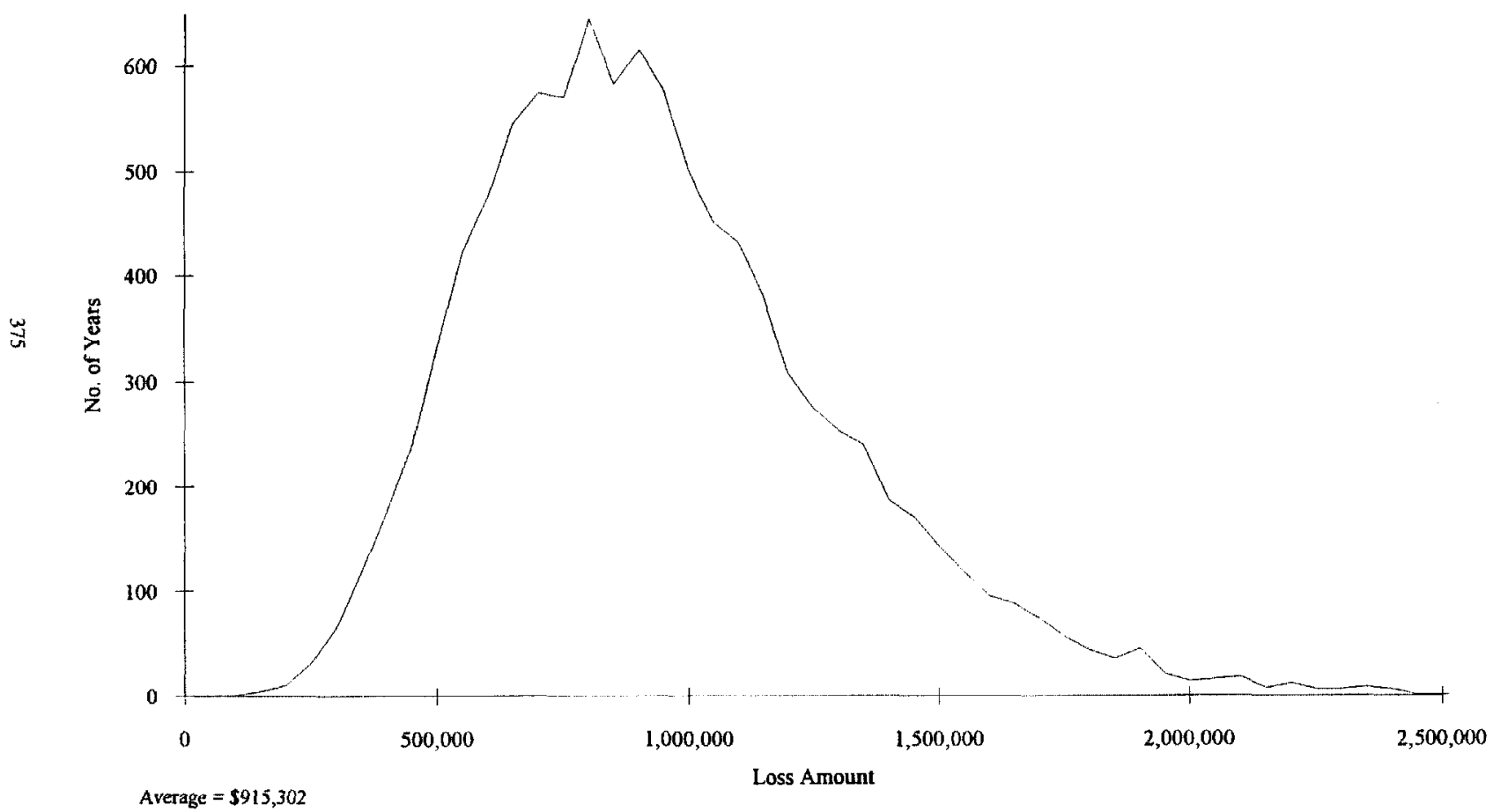


Exhibit B

Sheet 5

Aggregate IBNR Losses at Time $=4$

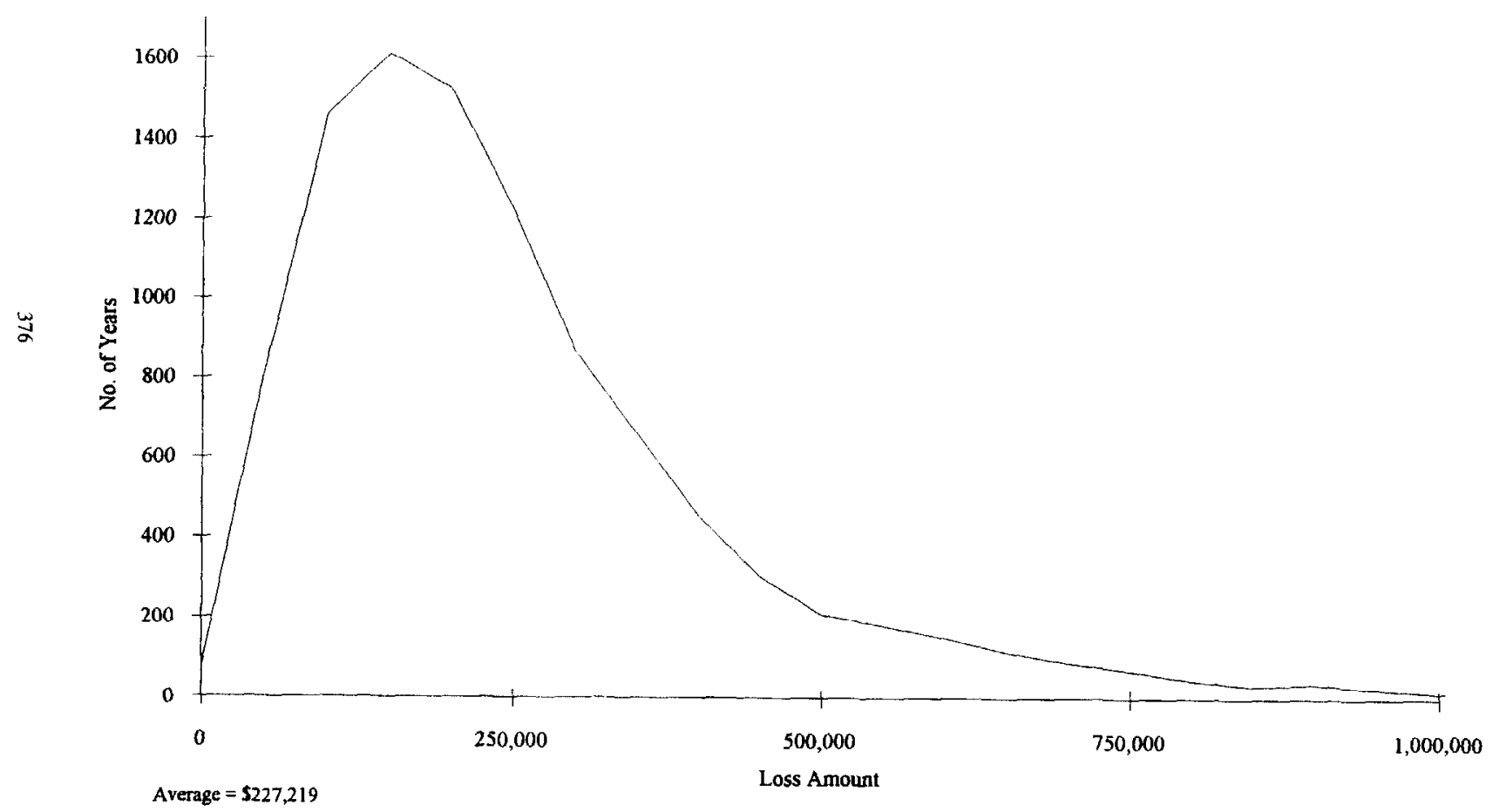


Exhibit C

\section{Incremental Paid Losses}

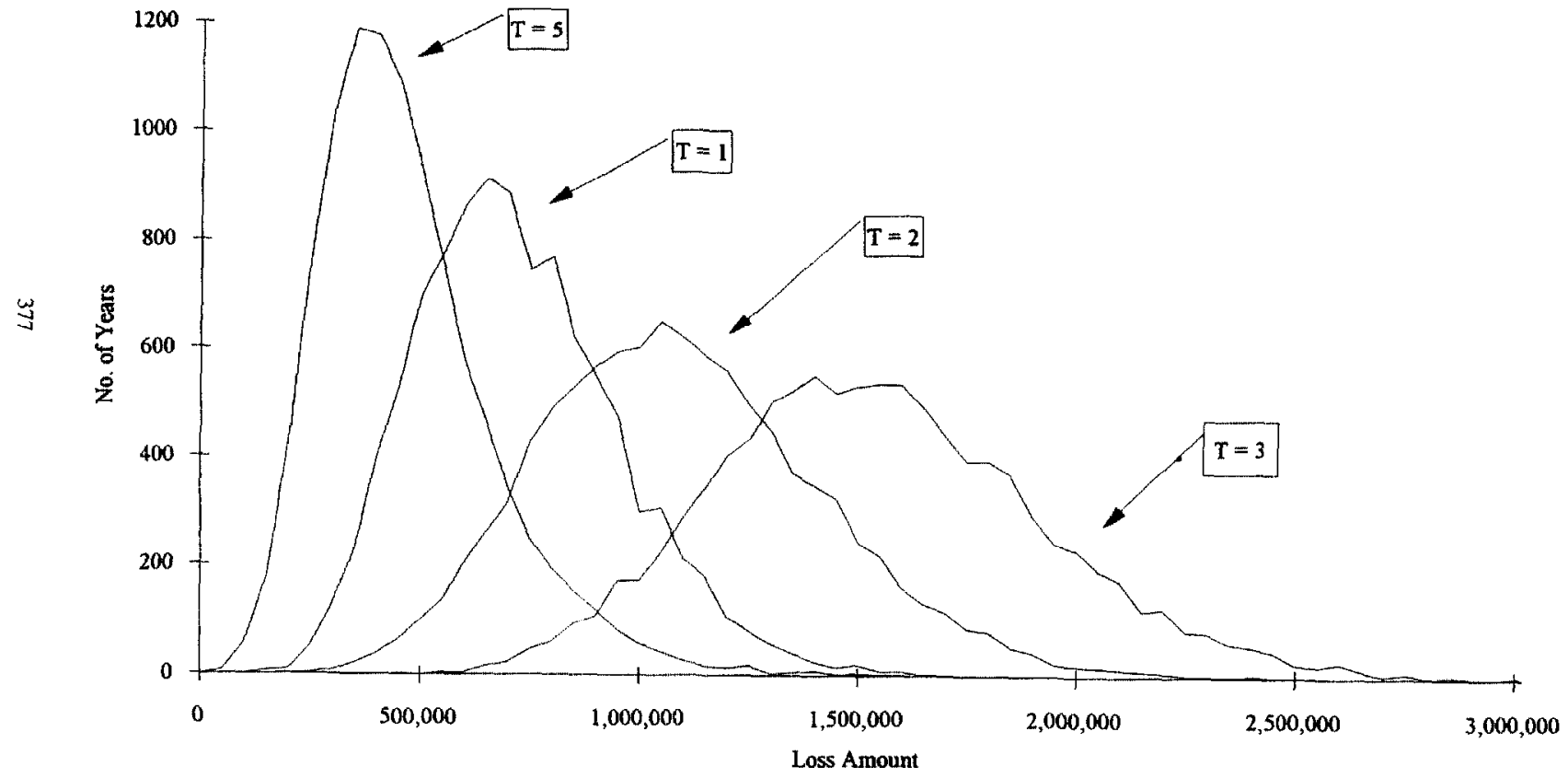


Incremental Incurred Losses

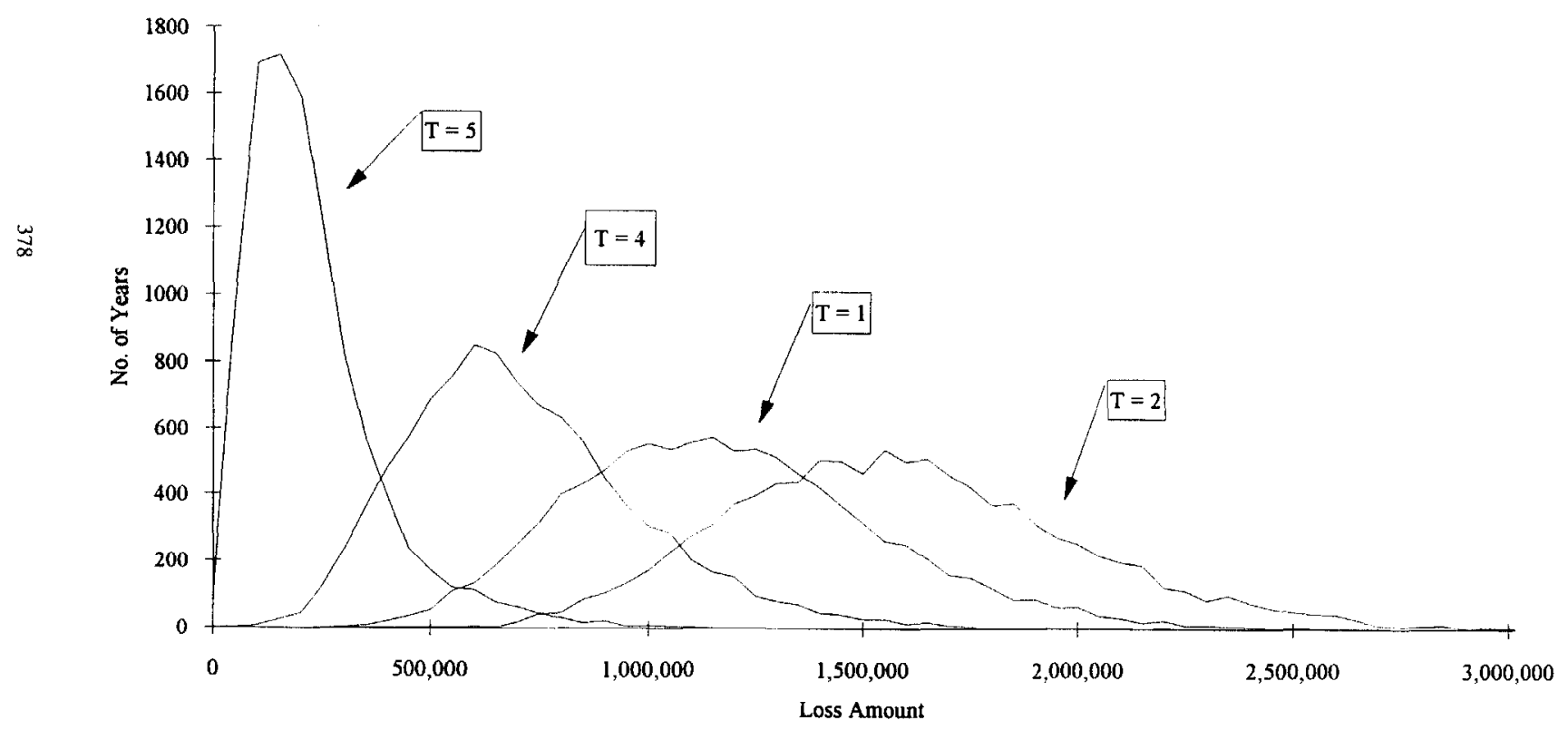




\section{Paid Loss Development Factors}

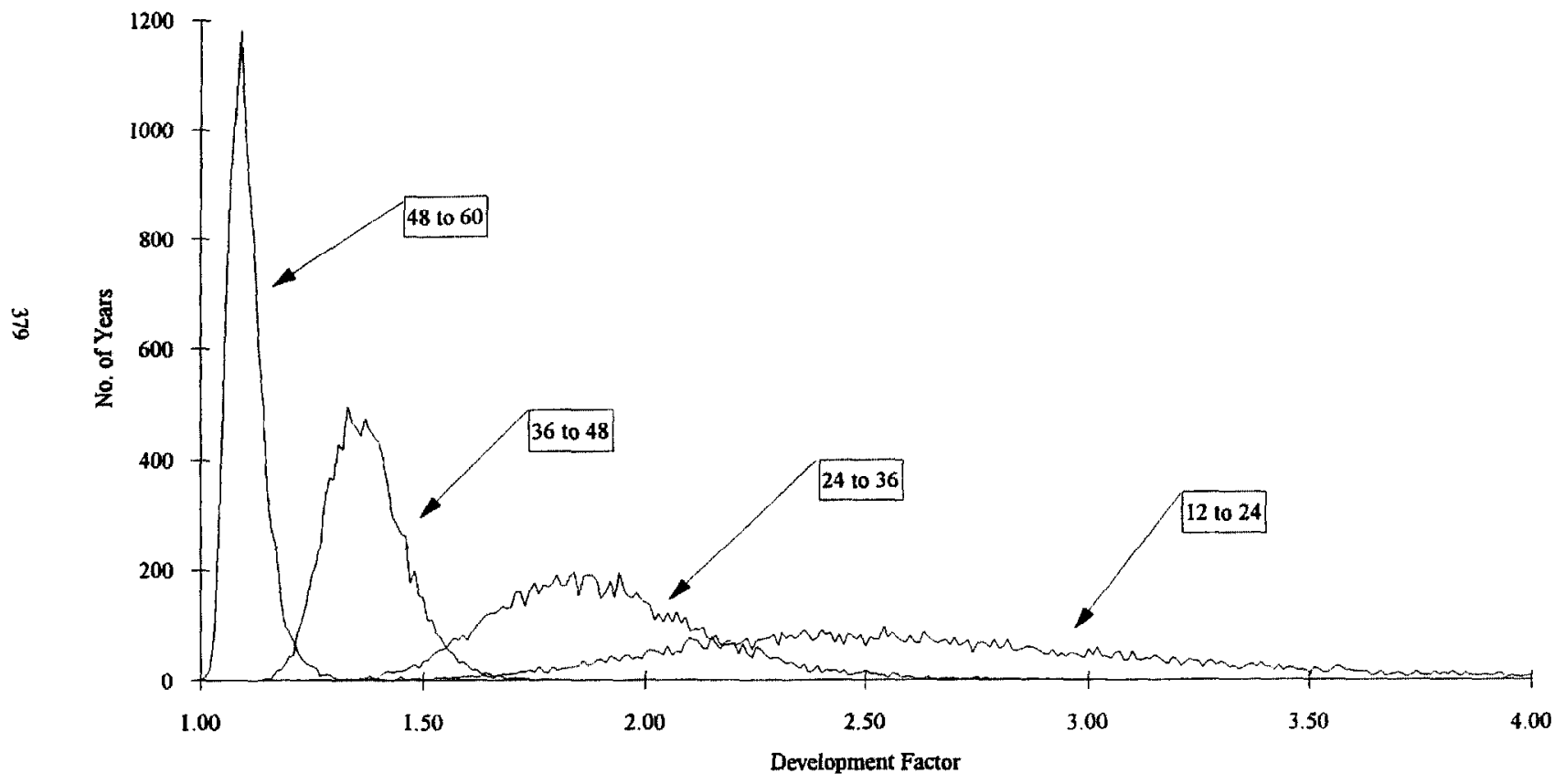


Paid Loss Development Factor - 12 to 24

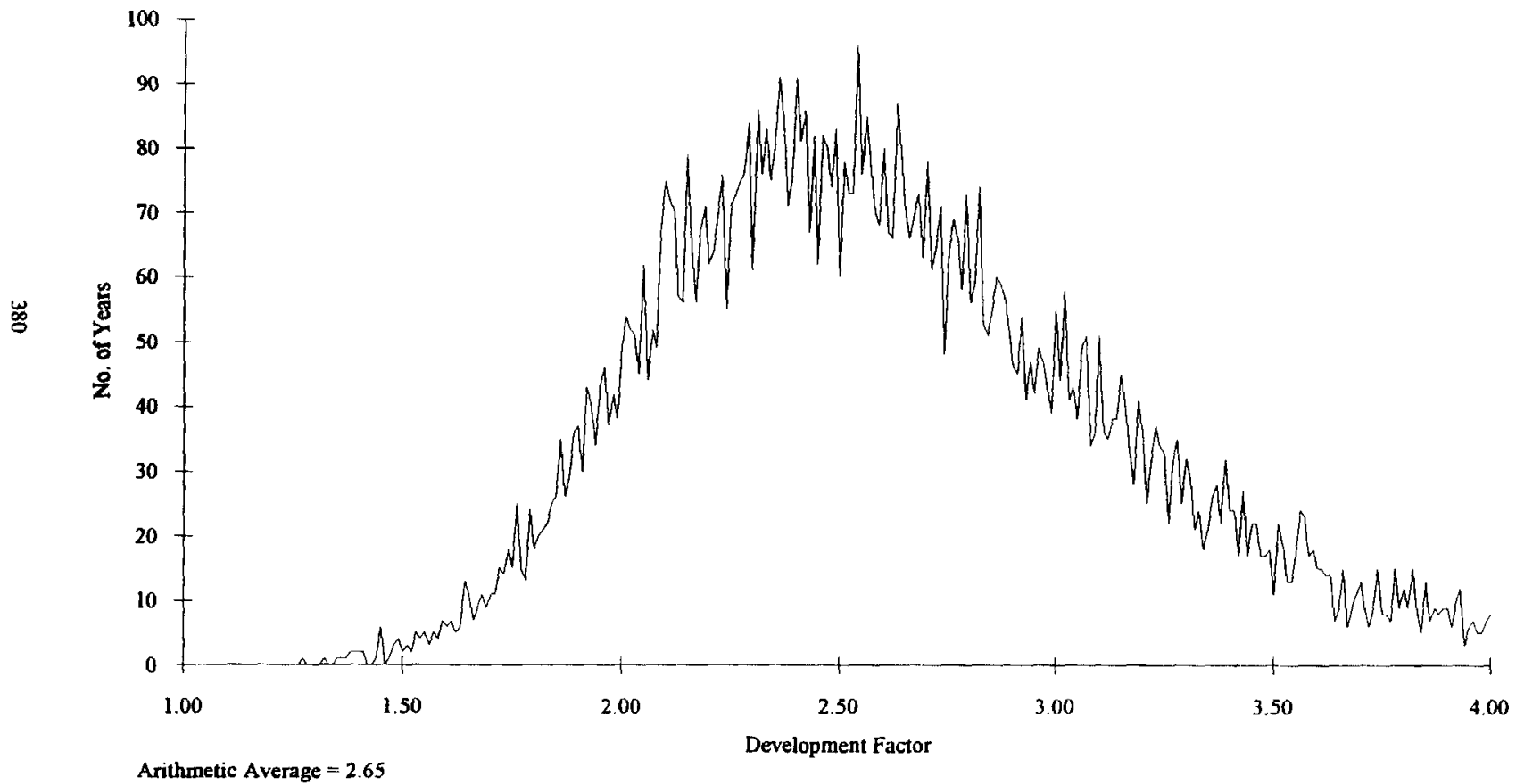


Paid Loss Development Factor - 24 to 36

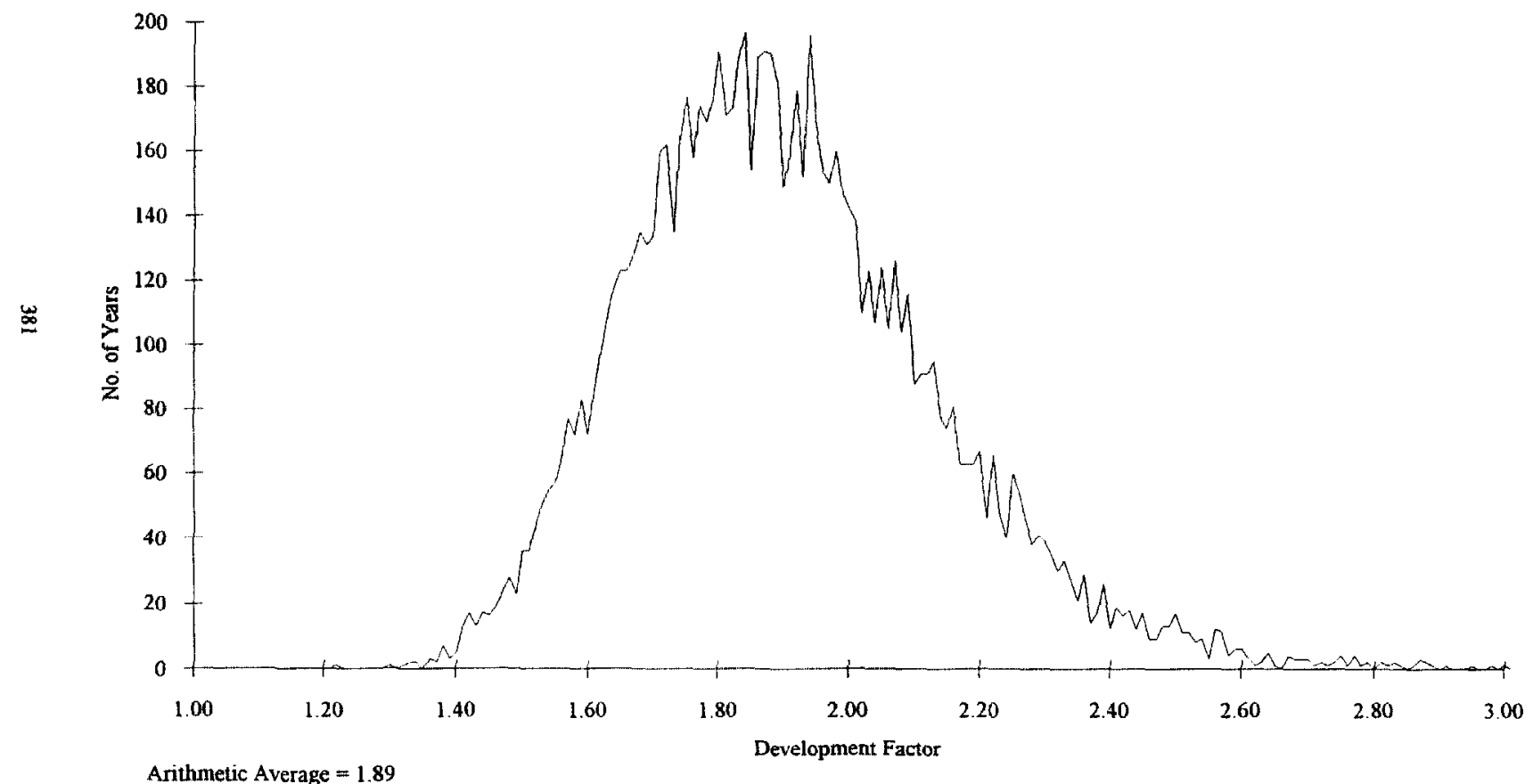


Exhibit E

Sheet 4

Paid Loss Development Factor - 36 to 48

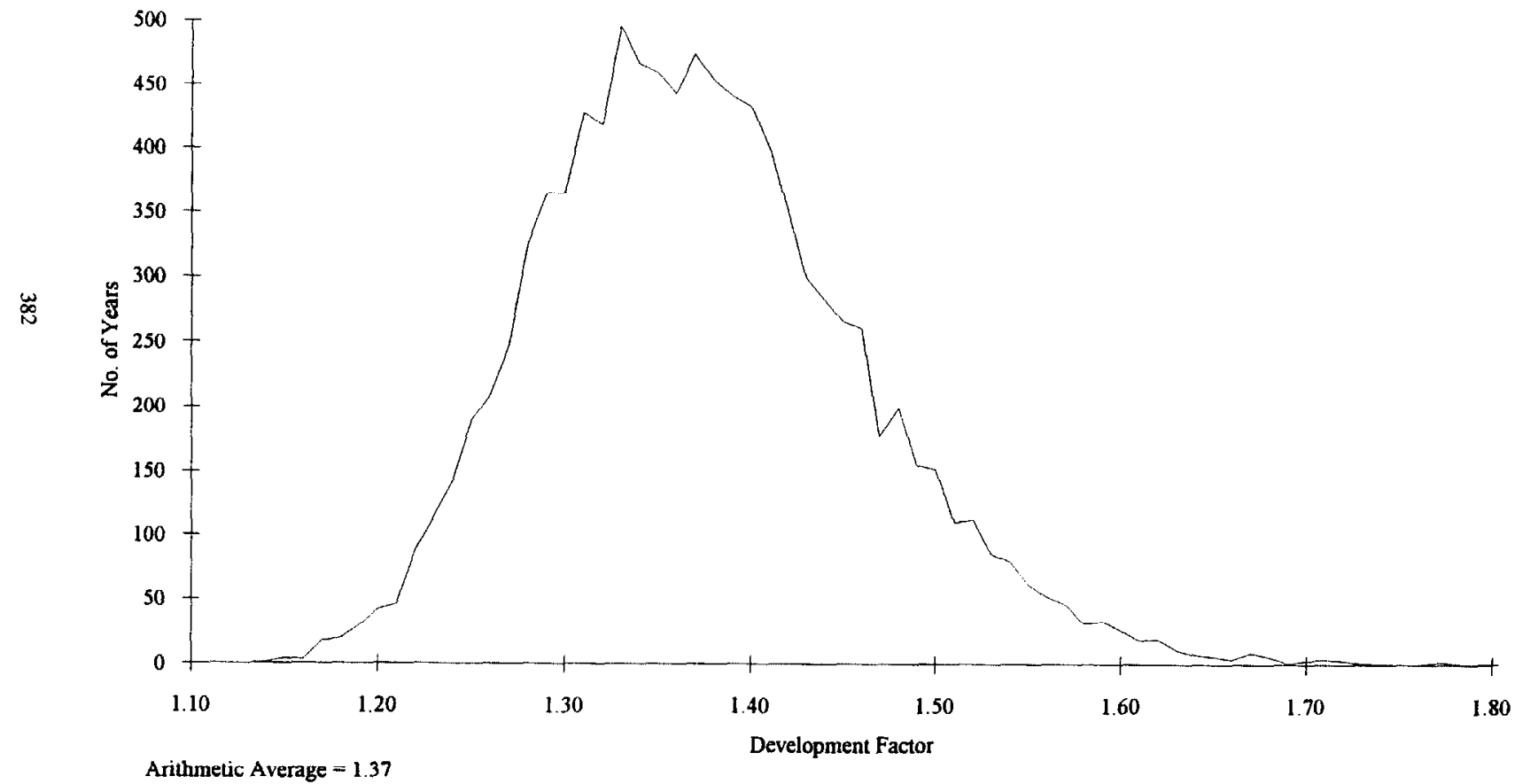


Exhibit F

Incurred Loss Development Factors

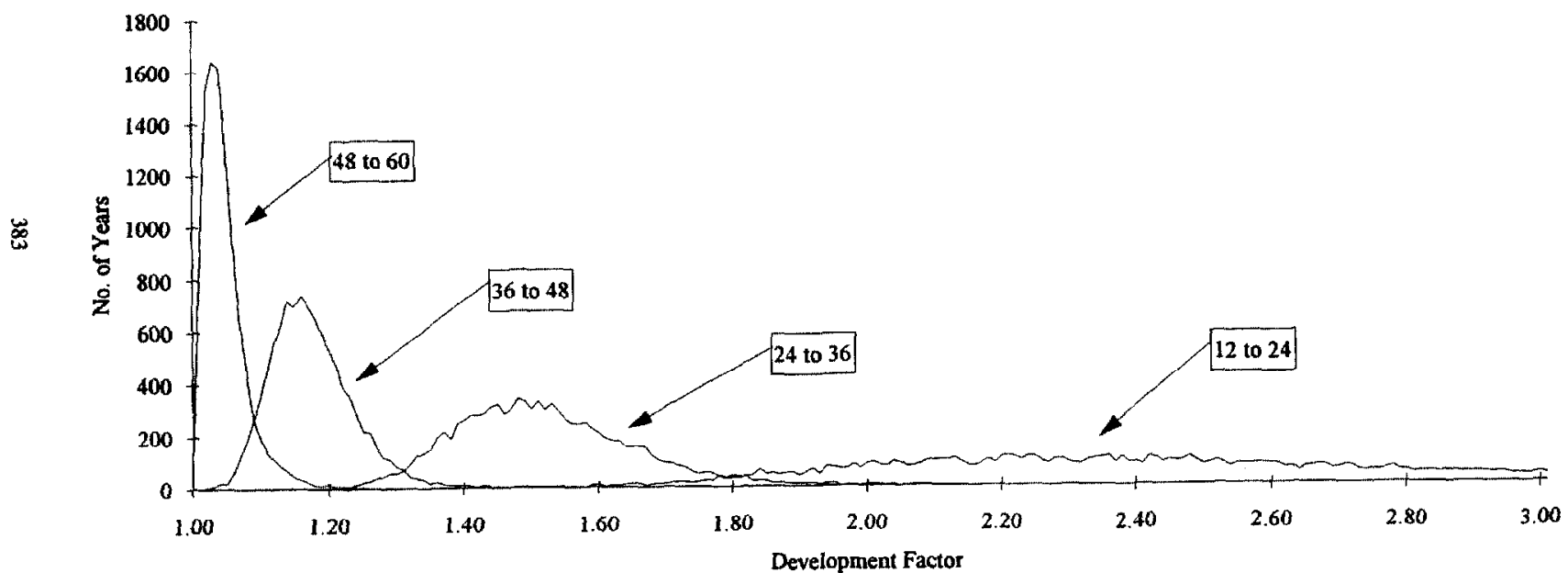


Run Off Ratio Analysis of Reserves at First Valuation

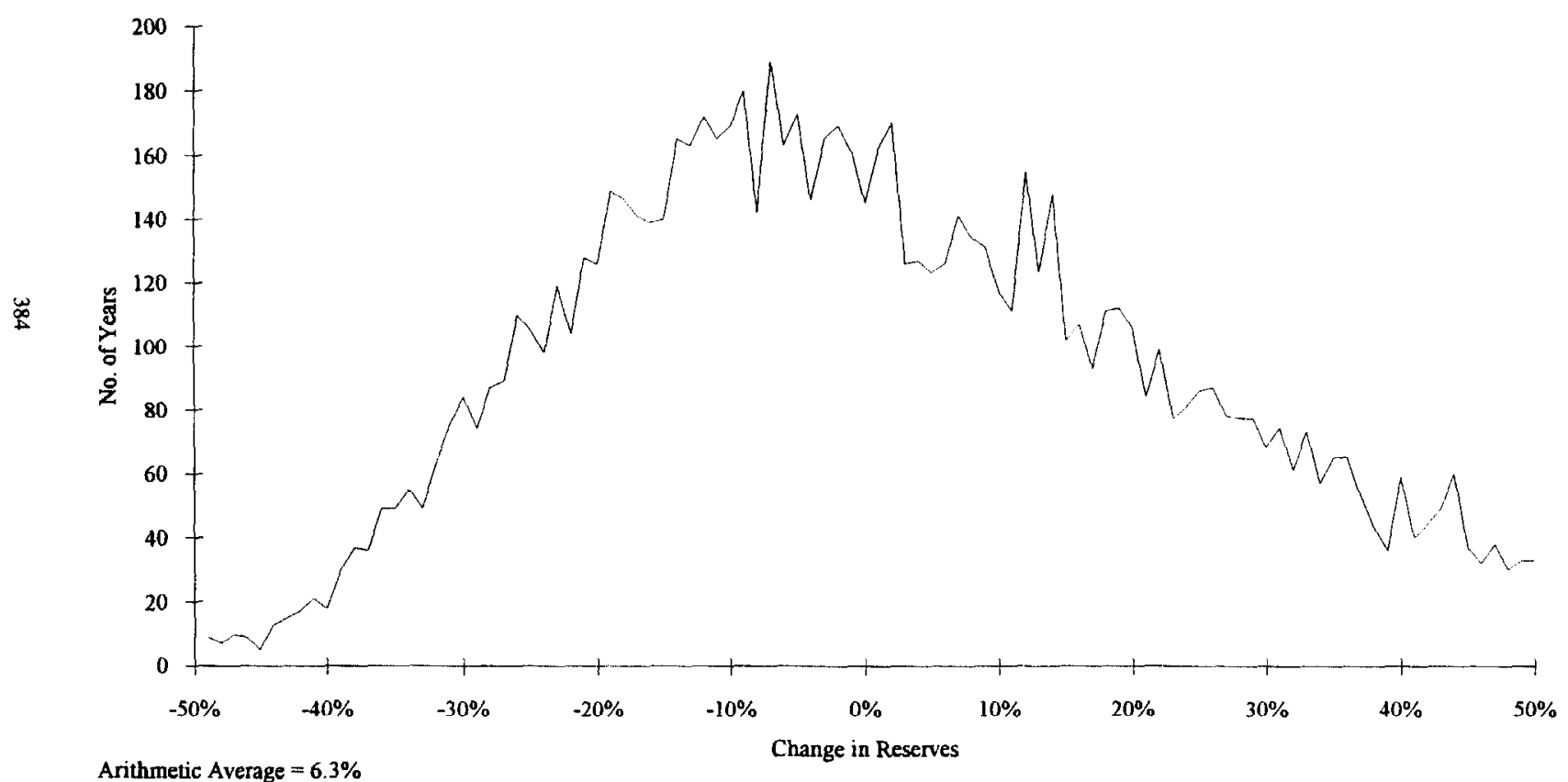


Appendix A

Sheet 1

Distribution of Case Incurred Losses




Case Incurred Loss Tranaition Matrix - 12 to 24

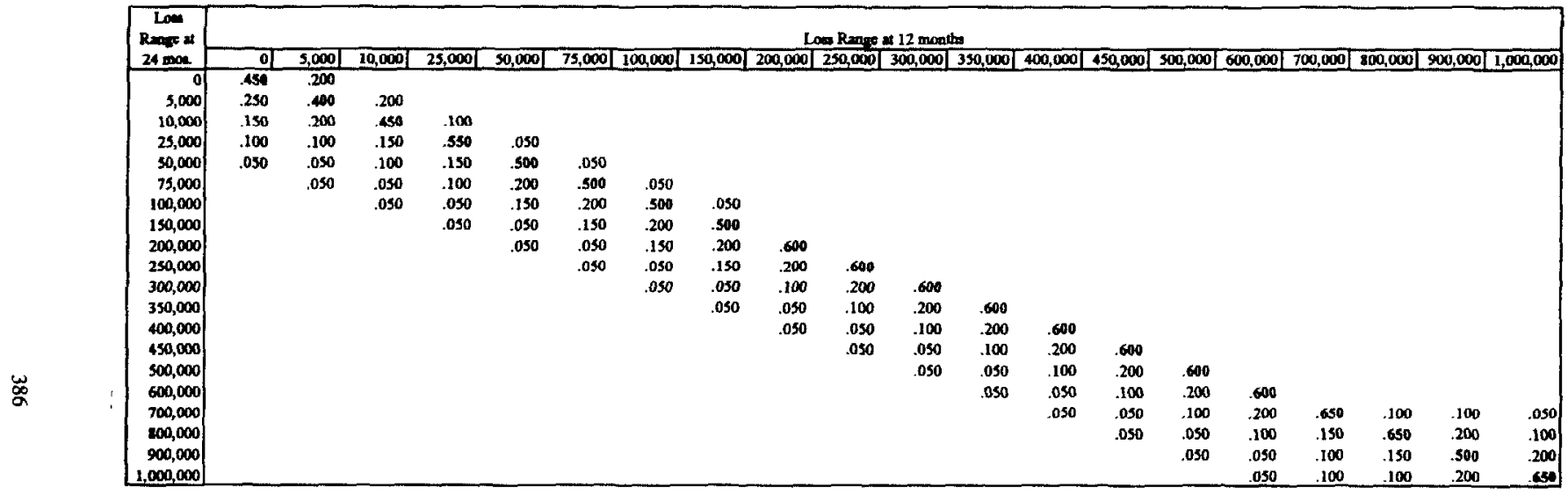




\begin{tabular}{|c|c|c|c|c|c|c|c|c|c|c|c|c|c|c|c|c|c|c|c|c|}
\hline \multirow{2}{*}{ 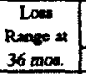 } & \multicolumn{20}{|c|}{ Lom Rugige it 24 month } \\
\hline & 可 & 5,000 & 10,000 & 25,000 & 50,000 & 75,000 & 100,000 & 150,000 & 200,000 & 250,000 & 300,000 & 350,000 & 400,000 & 450,000 & 500,0001 & 600,000 & 700,000 & 800,000 & 900,000 & $1,000,000$ \\
\hline & .700 & .100 & & & & & & & & & & & & & & & & & & \\
\hline 5,000 & .150 & .600 & .100 & & & & & & & & & & & & & & & & & \\
\hline 10,000 & .100 & .150 & .600 & .100 & & & & & & & & & & & & & & & & \\
\hline 25,000 & .050 & 100 & .150 & .600 & .100 & & & & & & & & & & & & & & & \\
\hline 50,000 & & .050 & .1000 & .150 & .600 & .100 & & & & & & & & & & & & & & \\
\hline 73,000 & & & .050 & .100 & .150 & .600 & & & & & & & & & & & & & & \\
\hline 100,000 & & & & .050 & .100 & .150 & .700 & & & & & & & & & & & & & \\
\hline 150,000 & & & & & .050 & .100 & .150 & 670 & & & & & & & & & & & & \\
\hline 200,000 & & & & & & .050 & .100 & .150 & .670 & & & & & & & & & & & \\
\hline 300,000 & & & & & & & & .050 & .100 & .150 & 550 & & & & & & & & & \\
\hline 350,000 & & & & & & & & .030 & .050 & .100 & .200 & .550 & & & & & & & & \\
\hline 400,000 & & & & & & & & & .030 & .050 & .100 & .200 & .550 & & & & & & & \\
\hline 450,000 & & & & & & & & & & .030 & .050 & .100 & 200 & .500 & & & & & & \\
\hline 500,000 & & & & & & & & & & .020 & .040 & .050 & .100 & .200 & .550 & & & & & \\
\hline 600,000 & & & & & & & & & & .010 & .030 & .040 & .050 & .100 & .200 & .600 & & & & \\
\hline 700,000 & & & & & & & & & & & .020 & .030 & .040 & .050 & .100 & .200 & .650 & .100 & 100 & 050 \\
\hline 200,000 & & & & & & & & & & & .010 & .020 & .030 & .050 & .050 & .100 & .130 & .650 & .200 & .100 \\
\hline 900,000 & & & & & & & & & & & & .010 & .020 & .050 & .050 & .050 & .100 & .150 & .500 & .200 \\
\hline 1.000 .000 & & & & & & & & & & & & & .010 & .050 & .050 & .050 & .100 & .100 & .200 & .650 \\
\hline
\end{tabular}


Appendix A

Sheat

Case Incurred Loss Transition Matrix - 36 to 48

\begin{tabular}{|c|c|c|c|c|c|c|c|c|c|c|c|c|c|c|c|c|c|c|c|c|}
\hline $\begin{array}{c}\text { Low } \\
\text { Renge } 14\end{array}$ & & & & & & & & & & ous Renge & $36 \mathrm{mont}$ & & & & & & & & & \\
\hline $48 \mathrm{mos}$. & 0 & 5,000 & 10,000 & 25,000 & 50,000 & 75,000 & 100,000 & 150,000 & 200,000 & 250,000 & 300,000 & 350,000 & 400,000 & 450,000 & 500,000 & 600,000 & 700,000 & 800,000 & $900,000 / 1,0$ & 000,000 \\
\hline & .900 & & & & & & & & & & & & & & & & & & & \\
\hline 3,000 & .100 & .950 & & & & & & & & & & & & & & & & & & \\
\hline 10,000 & & .050 & .950 & & & & & & & & & & & & & & & & & \\
\hline 25,000 & & & .030 & .950 & & & & & & & & & & & & & & & & \\
\hline 30,000 & & & & .050 & .950 & & & & & & & & & & & & & & & \\
\hline 75,000 & & & & & .050 & .950 & & & & & & & & & & & & & & \\
\hline 100,000 & & & & & & .050 & .950 & & & & & & & & & & & & & \\
\hline 150,000 & & & & & & & .050 & .850 & & & & & & & & & & & & \\
\hline 200,000 & & & & & & & & .100 & 850 & & & & & & & & & & & \\
\hline 250,000 & & & & & & & & .050 & 100 & .600 & & & & & & & & & & \\
\hline 300,000 & & & & & & & & & .050 & .150 & .500 & & & & & & & & & \\
\hline 350,000 & & & & & & & & & & .100 & .150 & .500 & & & & & & & & \\
\hline 400,000 & & & & & & & & & & .050 & .100 & .150 & .500 & & & & & & & \\
\hline 450,000 & & & & & & & & & & .050 & .100 & .100 & .150 & .500 & & & & & & \\
\hline 300,000 & & & & & & & & & & .030 & .050 & .050 & .100 & .200 & .500 & & & & & \\
\hline 600,000 & & & & & & & & & & .020 & .050 & .050 & .050 & .100 & .200 & .500 & & & & \\
\hline 700,000 & & & & & & & & & & & .050 & .050 & .050 & .050 & .100 & .200 & .600 & .050 & .050 & \\
\hline 800,000 & & & & & & & & & & & & .050 & .050 & .050 & .100 & .100 & .200 & .650 & .100 & .050 \\
\hline 900,000 & & & & & & & & & & & & .050 & .050 & .050 & .050 & .100 & .100 & .200 & .650 & .100 \\
\hline $1,000,000$ & & & & & & & & & & & & & .050 & .050 & .050 & .100 & .100 & .100 & .200 & 850 \\
\hline
\end{tabular}


Appendix A

Sheet 5

Distribution of Paid Losses

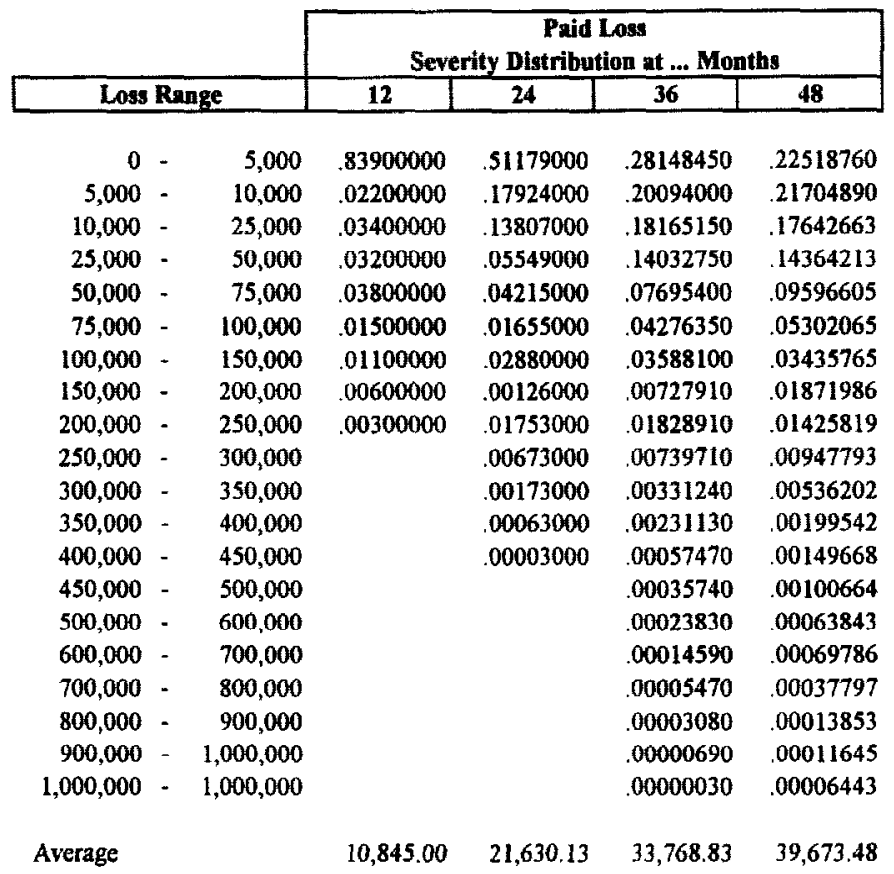


Appendux A Sheet 6

Paid Lose Tramaition Matrix - 12 to 24

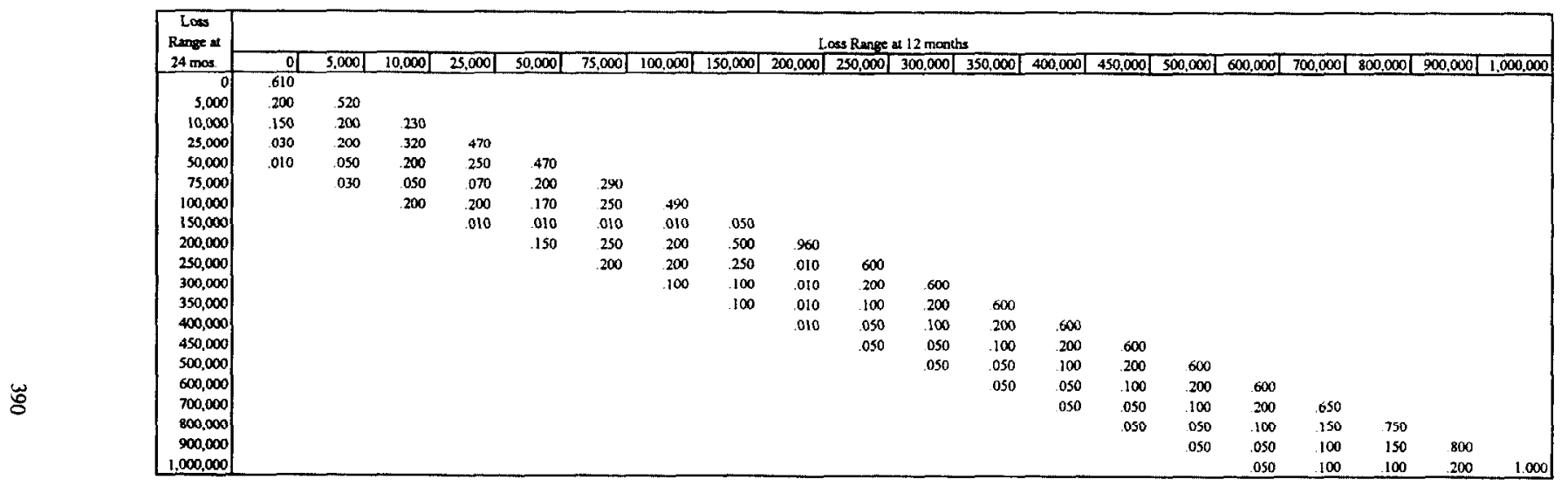


Paid Low Tranaition Mutrix - 24 to 36

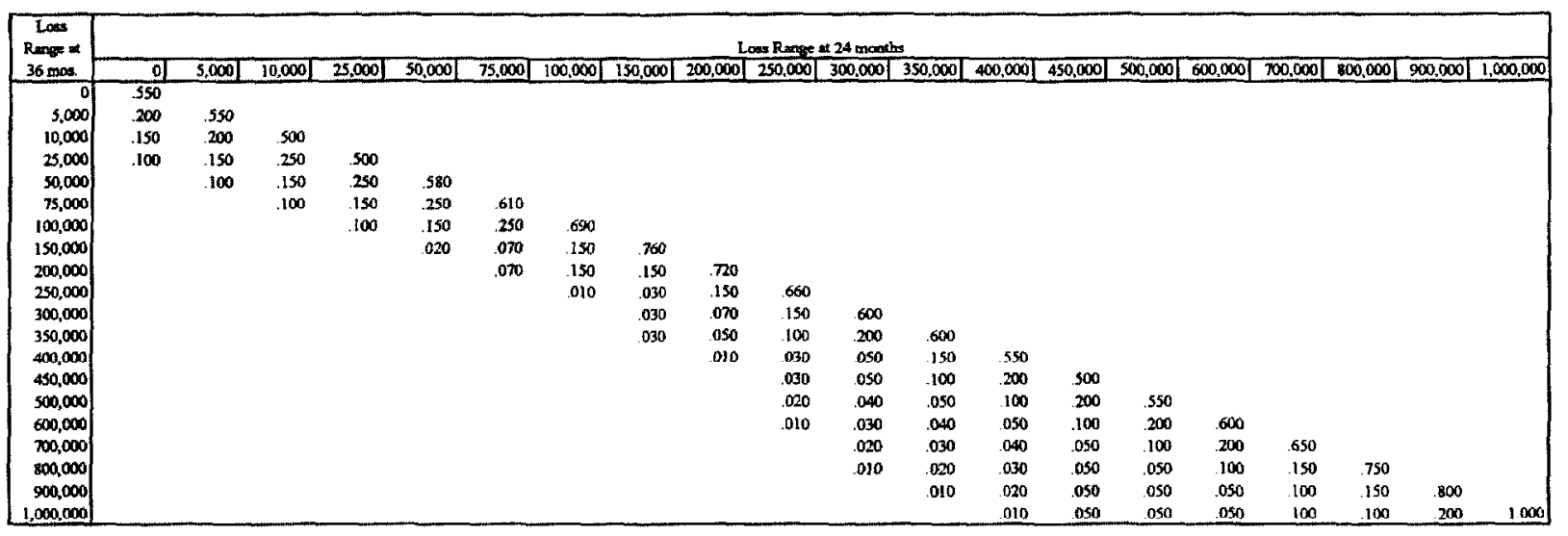


Appendix A Shear 8

Prid Low Tremeition Metrix - 36 to 48

\begin{tabular}{|c|c|c|c|c|c|c|c|c|c|c|c|c|c|c|c|c|c|c|c|c|}
\hline \multirow{2}{*}{$\begin{array}{c}\text { Loss } \\
\text { Rompex } \\
48 \text { inos } \\
\end{array}$} & \multicolumn{20}{|c|}{ Loss Ranes at 36 months } \\
\hline & 0 & 5,000 & 10,000 & 25,000 & 50,000 & 75,000 & 100,000 & 150,000 & 200,000 & 250,000 & 300,000 & 350,000 & $\$ 00,000$ & 450,000 & 500,000 & 600,000 & 700,000 & 800,000 & 900,000 & $1,000,000$ \\
\hline & 800 & & & & & & & & & & & & & & & & & & & \\
\hline 5,000 & .200 & .800 & & & & & & & & & & & & & & & & & & \\
\hline $10,0 \infty 0$ & & .200 & .750 & & & & & & & & & & & & & & & & & \\
\hline 25,000 & & & .250 & .700 & & & & & & & & & & & & & & & & \\
\hline 50,000 & & & & .300 & .700 & & & & & & & & & & & & & & & \\
\hline 75,000 & & & & & .300 & .700 & & & & & & & & & & & & & & \\
\hline 100,000 & & & & & & .300 & .600 & & & & & & & & & & & & & \\
\hline 150,000 & & & & & & & .400 & .600 & & & & & & & & & & & & \\
\hline 200,000 & & & & & & & & .200 & .700 & & & & & & & & & & & \\
\hline 250,000 & & & & & & & & .200 & .200 & .590 & & & & & & & & & & \\
\hline 300,000 & & & & & & & & & .100 & 200 & 620 & & & & & & & & & \\
\hline 350,000 & & & & & & & & & & .050 & .100 & .560 & & & & & & & & \\
\hline 400,000 & & & & & & & & & & .050 & .100 & .200 & 580 & & & & & & & \\
\hline 450,000 & & & & & & & & & & .050 & .050 & .100 & 200 & .350 & & & & & & \\
\hline 500,000 & & & & & & & & & & .030 & .030 & .040 & .080 & .200 & .450 & & & & & \\
\hline 600,000 & & & & & & & & & & .030 & .050 & .050 & .050 & .100 & .210 & .550 & & & & \\
\hline 700,000 & & & & & & & & & & & .050 & .030 & .050 & .100 & .100 & .150 & .600 & & & \\
\hline 800,000 & & & & & & & & & & & & .010 & .020 & .100 & .100 & .100 & 150 & .200 & & \\
\hline 900,000 & & & & & & & & & & & & .010 & .010 & .100 & .070 & .100 & .150 & .200 & .900 & \\
\hline $1,000,000$ & & & & & & & & & & & & & .010 & .050 & .070 & .100 & .100 & .100 & .100 & 1.000 \\
\hline
\end{tabular}

